\title{
NOTE ON ARC-LENGTH AND HARMONIC MEASURE
}

\author{
JOHN S. SPRAKER
}

(Communicated by John B. Conway)

\begin{abstract}
In this note it is shown that the only Smirnov domain for which arc-length measure is harmonic measure is the disk. The proof depends on some facts about inner and outer functions and arc-length preserving maps.
\end{abstract}

A domain $R$ bounded by a rectifiable Jordan curve is called Smirnov if the derivative of the conformal map which takes the unit disk onto $R$ is an outer function. Examples include starlike domains and domains with analytic boundary. Smirnov tried for some time to show that all rectifiable Jordan domains were Smirnov domains. Finally Keldysh and Lavrentiev in [3] constructed a pathological counterexample. For a somewhat simpler construction see $[1,2$, or 4].

In what follows let $D$ be the unit disk, let $\Lambda_{1}$ denote arc-length measure, and let $\omega_{a}$ denote harmonic measure for $R$ at the point $a$.

Theorem. If $\psi: R \rightarrow G$ is a conformal arc-length preserving map between two Smirnov domains $R$ and $G$, the $\psi$ is linear with $\left|\psi^{\prime}\right|=1$.

Proof. Suppose $\phi: D \rightarrow R$ is conformal. Then since $R$ and $G$ are Smirnov domains $\psi^{\prime}(\phi(z)) \phi^{\prime}(z)$ and $\phi^{\prime}(z)$ must be outer functions. Thus $\psi^{\prime}(\phi(z))=$ $\psi^{\prime}(\phi(z)) \phi^{\prime}(z) / \phi^{\prime}(z)$ is also seen to be outer by considering the integral representation of an outer function. Let $E$ be a Borel subset of $\partial G$. Then

$$
\begin{aligned}
\int_{\phi^{-1}\left(\psi^{-1}(E)\right)}\left|\psi^{\prime}(\phi(z)) \| \phi^{\prime}(z)\right| d \Lambda_{1}(z) & =\Lambda_{1}(E) \\
& =\Lambda_{1}\left(\psi^{-1}(E)\right) \\
& \text { since } \psi \text { preserves arc-length } \\
& =\int_{\phi^{-1}\left(\psi^{-1}(E)\right)}\left|\phi^{\prime}(z)\right| d \Lambda_{1}
\end{aligned}
$$

Received by the editors August 19, 1988.

1980 Mathematics Subject Classification (1985 Revision). Primary 30C85; Secondary 30C35, $31 \mathrm{~A} 15$.

Key words and phrases. Inner functions, outer functions, Smirnov domains, harmonic measure, arc-length measure.

The contents of this paper were presented to the Kentucky Academy of Science at its annual meeting on November 5, 1988. 
Since this holds for all Borel sets $E$ we have that $\left|\psi^{\prime}(\phi(z))\right|=1$ a.e. on $\partial D$. Thus $\psi^{\prime}(\phi(z))$ is both an inner and an outer function and therefore constant. The result follows.

The previous theorem shows that an arc-length preserving conformal map changes only the position and orientation of a Smirnov domain not its shape.

Corollary. The only Smirnov domain such that $(1 / 2 \pi) \Lambda_{1}=\omega_{a}$ is the unit disk centered at $a$.

Proof. Let $\omega_{a}=(1 / 2 \pi) \Lambda_{1}$ for a Smirnov domain $R$ and let $\phi: D \rightarrow R$ be a conformal map which takes 0 to $a$. Since conformal maps carry harmonic measure to harmonic measure and the harmonic measure for $D$ at 0 is arclength measure, $\phi$ is an arc-length preserving map. Thus $\phi$ is linear by the previous result and $\phi^{\prime}$ is a constant of modulus one, so $\phi(D)$ is the unit disk centered at $a$.

Note. The hypothesis that $R$ be Smirnov cannot be removed as examples in [1, 2, 3, and 4] show.

\section{REFERENCES}

1. P. L. Duren, Theory of $H^{p}$ space, Academic Press, New York, 1970.

2. P. L. Duren, H. S. Shapiro and A. L. Shields, Singular measures and domains not of Smirnov type, Duke Math. J. 33 (1966), 247-254.

3. M. V. Keldysh and M. A. Lavrentiev, Sur la representation conforme des domains limites par des courbes rectifiables, Ann. Sci. École Norm. Sup. 54 (1937), 1-38.

4. G. Piranian, Two monotonic, singular uniformly almost smooth functions, Duke Math. J. 33 (1966), 255-262.

Department of Mathematics, Western Kentucky University, Bowling Green, KenTUCKY 42101 